\title{
Revealing unconventional host-guest complexation at nanostructured interface by surface-enhanced Raman spectroscopy
}

\author{
Gan-Yu Chen', Yi-Bin Sun', Pei-Chen Shi', Tao Liu², Zhi-Hao Li', Si-Heng Luo ${ }^{1,2}$, Xin-Chang Wang ${ }^{3}$, Xiao-Yu Cao ${ }^{1,4}$,

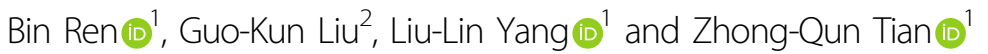

\begin{abstract}
Interfacial host-guest complexation offers a versatile way to functionalize nanomaterials. However, the complicated interfacial environment and trace amounts of components present at the interface make the study of interfacial complexation very difficult. Herein, taking the advantages of near-single-molecule level sensitivity and molecular fingerprint of surface-enhanced Raman spectroscopy (SERS), we reveal that a cooperative effect between cucurbit[7] uril (CB[7]) and methyl viologen $\left(\mathrm{MV}^{2+} 2 \mathrm{I}^{-}\right)$in aggregating Au NPs originates from the cooperative adsorption of halide counter anions $\mathrm{I}^{-}, \mathrm{MV}^{2+}$, and $\mathrm{CB}[7]$ on Au NPs surface. Moreover, similar SERS peak shifts in the control experiments using $\mathrm{CB}[\mathrm{n}] \mathrm{s}$ but with smaller cavity sizes suggested the occurrence of the same guest complexations among $\mathrm{CB}[5], \mathrm{CB}$ [6], and $\mathrm{CB}[7]$ with $\mathrm{MV}^{2+}$. Hence, an unconventional exclusive complexation model is proposed between CB[7] and $\mathrm{MV}^{2+}$ on the surface of Au NPs, distinct from the well-known 1:1 inclusion complexation model in aqueous solutions. In summary, new insights into the fundamental understanding of host-guest interactions at nanostructured interfaces were obtained by SERS, which might be useful for applications related to host-guest chemistry in engineered nanomaterials.
\end{abstract}

\section{Introduction}

Host-guest chemistry offers a reversible and versatile way to achieve high-fidelity recognition between host and guest molecules; therefore, it is widely employed in homogeneous solutions ${ }^{1-4}$. In recent studies, host-guest systems have been used on solid surfaces to functionalize nanomaterials ${ }^{5-12}$. The results showed that the host-guest complexation behavior on surfaces may differ from those observed in solutions. For instance, host-guest

\section{Correspondence: Guo-Kun Liu (guokunliu@xmu.edu.cn) or Liu-}

Lin Yang (llyang@xmu.edu.cn) or Zhong-Qun Tian (zqtian@xmu.edu.cn) ${ }^{1}$ State Key Laboratory of Physical Chemistry of Solid Surfaces, Collaborative Innovation Center of Chemistry for Energy Materials (iChEM), College of Chemistry and Chemical Engineering, Xiamen University, Xiamen 361005, China

${ }^{2}$ State Key Laboratory of Marine Environmental Science, Fujian Provincial Key Laboratory for Coastal Ecology and Environmental Studies, Center for Marine Environmental Chemistry \& Toxicology, College of the Environment and Ecology, Xiamen University, Xiamen 361102, China

Full list of author information is available at the end of the article complexation on surfaces may greatly enhance compared with solutions. This can be explained by the preorganization of ligands on surfaces, as well as the synergistic effects induced by multiple noncovalent interactions ${ }^{5}$. Besides, competitive adsorptions on surfaces may lead to unpredictable surface environments, thereby affecting the interfacial host-guest complexation. As a result, it is important to better understand the interfacial behavior of host-guest systems for their wider and more robust applications in nanomaterials. However, the complex surface effects and trace amounts of components present on the surface make the study of interfacial complexation more difficult ${ }^{13}$.

Several techniques such as extinction spectroscopy, fluorescence spectroscopy ${ }^{14-16}$, and cyclic voltammetry ${ }^{17,18}$ have been used to monitor the host-guest complexation at interfaces. However, these methods often require the use of chromophores or redox-active

\section{(c) The Author(s) 2021}

(c) (i) Open Access This article is licensed under a Creative Commons Attribution 4.0 International License, which permits use, sharing, adaptation, distribution and reproduction cc) in any medium or format, as long as you give appropriate credit to the original author(s) and the source, provide a link to the Creative Commons license, and indicate if changes were made. The images or other third party material in this article are included in the article's Creative Commons license, unless indicated otherwise in a credit line to the material. If material is not included in the article's Creative Commons license and your intended use is not permitted by statutory regulation or exceeds the permitted use, you will need to obtain permission directly from the copyright holder. To view a copy of this license, visit http://creativecommons.org/licenses/by/4.0/. 
motifs and suffer from background interferences generated by nanoparticles (NPs). Nuclear magnetic resonance (NMR) spectroscopy provides information about ligand-shell morphology or ligand exchange on metal $\mathrm{NPs}^{19-21}$, but suffers from relatively high detection limit and background interference caused by residual agents in solutions. Alternatively, surface-enhanced Raman spectroscopy (SERS) provides rich molecular vibrational information along with many advantages in terms of ultrahigh sensitivity, label-free and in situ detection, distinction among ligand types, and exclusive detection of adsorbates present on the surface ${ }^{22,23}$. Furthermore, SERS peaks of host and/or guest could shift upon host-guest complexation ${ }^{13,24-27}$, beneficial for investigating host-guest complexation at the interface. Therefore, SERS is a practical and powerful in situ technique for revealing interfacial host-guest complexation phenomena.

In this study, SERS was used to investigate host-guest interactions between cucurbit[7]uril (CB[7]) and methyl viologen $\left(\mathrm{MV}^{2+} 2 \mathrm{I}^{-}\right)$at $\mathrm{Au} \mathrm{NP}$-water interface (Fig. 1a). SERS studies showed that a cooperative effect between $\mathrm{CB}$ [7] and $\mathrm{MV}^{2+} 2 \mathrm{I}^{-}$in regulating the aggregation of Au NPs originated from the cooperative adsorption of halide counter anions $\mathrm{I}^{-}, \mathrm{MV}^{2+}$, and $\mathrm{CB}[7]$ on $\mathrm{Au} \mathrm{NP}$ surface. By carefully analyzing the SERS spectra at different molar ratios of host and guest as well as the Raman spectra in homogeneous solutions, we deduced that the complexation between $\mathrm{MV}^{2+}$ and $\mathrm{CB}[7]$ at the interface is different from that in solutions. Moreover, similar SERS peak shifts in the control experiments using $\mathrm{CB}[\mathrm{n}] \mathrm{s}$ but with different cavity sizes suggested the occurrence of same guest complexations among $\mathrm{CB}[5], \mathrm{CB}[6]$, and $\mathrm{CB}[7]$. Hence, we propose an unconventional exclusive complexation model between $\mathrm{CB}$ [7] and $\mathrm{MV}^{2+}$ on the surface of Au NPs which is distinct from the well-known 1:1 inclusion complexation model (Fig. S1) in aqueous solutions ${ }^{28,29}$.

\section{Results \\ Cooperative effect of host and guest on Au NPs aggregation}

In this study, a mixed solution of $\mathrm{CB}$ [7] $(0.31 \mu \mathrm{M})$ and $\mathrm{MV}^{2+} 2 \mathrm{I}^{-}(2.5 \mu \mathrm{M})$ induced an instant aggregation of $\mathrm{Au}$ NPs (Fig. 1b). The time-dependent extinction spectra revealed a drastic decline in the localized surface plasmon resonance (LSPR) peak of Au NPs (ca. $526 \mathrm{~nm}$ ) within $1 \mathrm{~min}$ (Fig. 1b), accompanied by the generation of a new LSPR peak above $800 \mathrm{~nm}$. After $4 \mathrm{~min}$, the spectrum remained almost unchanged, suggesting that the aggregation of Au NPs was almost completed within $1 \mathrm{~min}$. In comparison, $\mathrm{CB}[7]$ or $\mathrm{MV}^{2+} 2 \mathrm{I}^{-}$alone failed to produce the aggregation of colloidal $\mathrm{Au}$ at the same concentration within 5 min (Fig. S2). Though $\mathrm{CB}[7]$ alone at a higher concentration can also aggregate $\mathrm{Au} \mathrm{NPs}{ }^{30,31}$, the process a<smiles>C[n+]1ccc(-c2cc[n+](C)cc2)cc1</smiles>

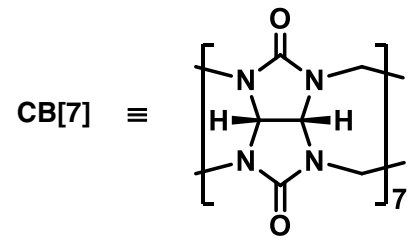

\section{b}

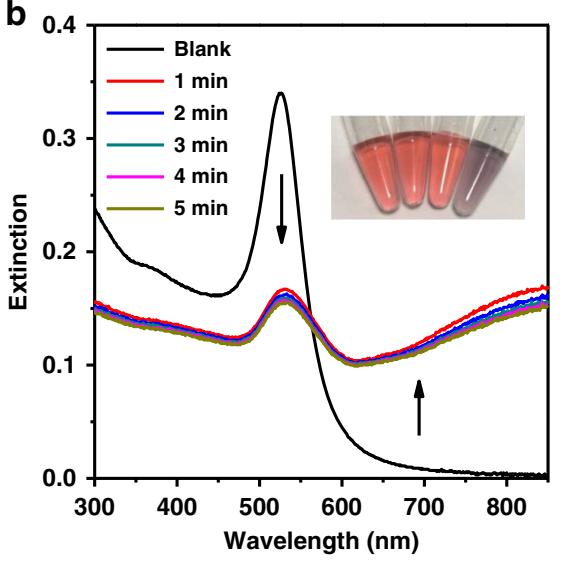

C

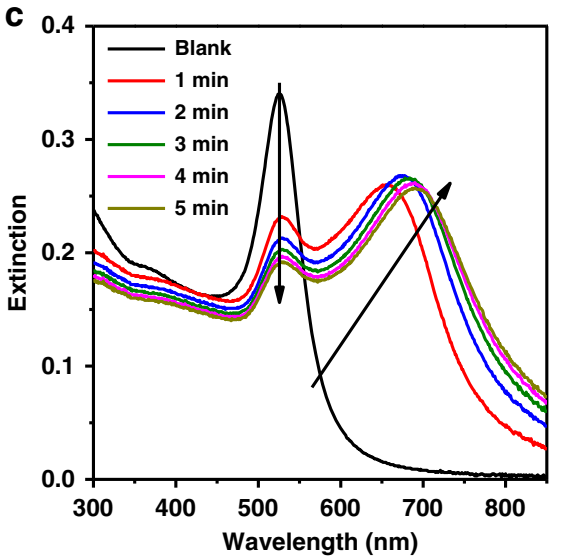

Fig. 1 Host-guest solution induced aggregation of Au NPs. a Molecular structures of $\mathrm{MV}^{2+} 2 \mathrm{I}^{-}$and $\mathrm{CB}[7]$. $\mathbf{b}$ Time-dependent extinction spectra of Au NPs colloid obtained by adding $\mathrm{MV}^{2+} 21^{-}$and $\mathrm{CB}[7]$. Inset shows the corresponding photographs of Au NPs colloid (from left to right) on the addition of $\mathrm{H}_{2} \mathrm{O}, \mathrm{MV}^{2+} 2 \mathrm{I}^{-}, \mathrm{CB}[7]$, and $\mathrm{MV}^{2+} 2 \mathrm{I}^{-}+\mathrm{CB}[7]$, respectively. The concentrations of $\mathrm{MV}^{2+} 2 \mathrm{I}^{-}$and $\mathrm{CB}[7]$ are $2.5 \mu \mathrm{M}$ and $0.31 \mu \mathrm{M}$, respectively. $\mathbf{c}$ Time-dependent extinction spectra of Au NPs colloid on the addition of $250 \mu \mathrm{M}$ CB[7] 

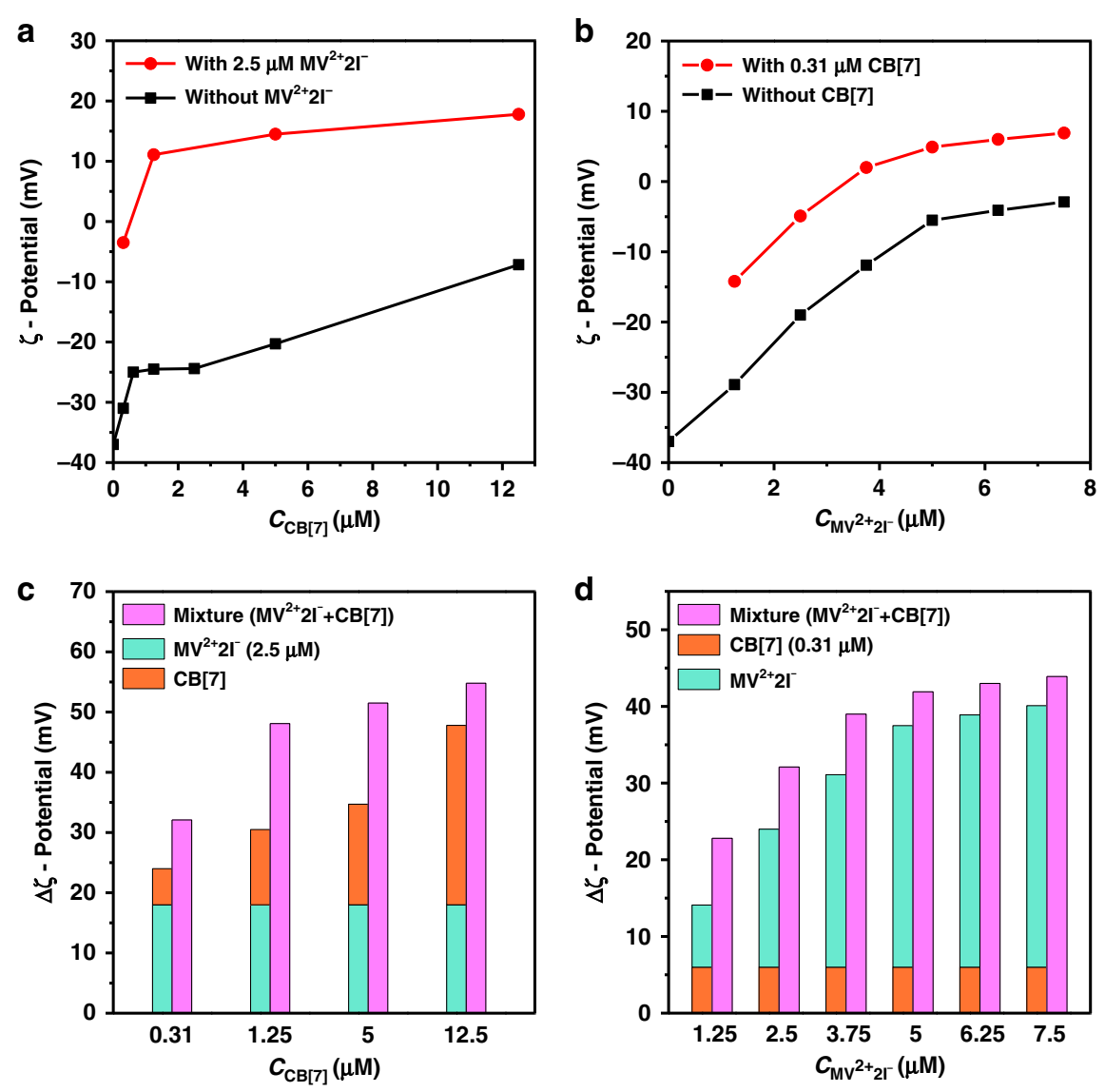

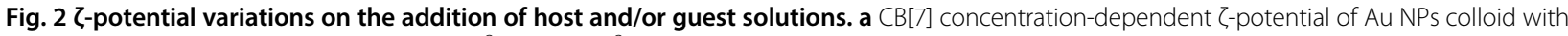
(red line) and without (black line) $2.5 \mu \mathrm{M} \mathrm{MV}^{2+} 2 \mathrm{I}^{-}$. $\mathbf{b ~ M V ^ { 2 + }} 2 \mathrm{I}^{-}$concentration-dependent $\zeta$-potential of Au NPs colloid with (red line) and without (black line) $0.31 \mu \mathrm{M} \mathrm{CB}[7]$. $\mathbf{c}$ and $\mathbf{d}$ Pink columns show the variations in $\zeta$-potential $\left(\Delta \zeta_{\text {mix }}\right.$ ) with simultaneous additions of $\mathrm{CB}[7]$ and $\mathrm{MV}^{2+} 2 \mathrm{I}^{-}$. Orange and green columns display the sum in variations of $\zeta$-potential $\left(\Delta \zeta_{\text {sum }}\right)$ caused solely by $\mathrm{CB}[7]$ and $\mathrm{MV}^{2+} 2 \mathrm{I}^{-}$

was much less efficient (Fig. 1c). The much faster and efficient aggregation of $\mathrm{Au}$ NPs in the presence of $\mathrm{CB}[7]$ and $\mathrm{MV}^{2+} 2 \mathrm{I}^{-}$suggested a cooperative effect of host and guest molecules in aggregating Au NPs.

The cooperative effect of host and guest molecules in aggregating Au NPs was further confirmed from the variations in $\zeta$-potential. To this end, two sets of $\zeta$ potential measurements were carried out. The first $\zeta$ potential was recorded as a function of the concentration of $\mathrm{CB}[7]$ with and without the addition of $\mathrm{MV}^{2+} 2 \mathrm{I}^{-}$. The second $\zeta$-potential was recorded as a function of $\mathrm{MV}^{2+} 2 \mathrm{I}^{-}$ concentration with and without $\mathrm{CB}[7]$. As shown in Fig. $2 \mathrm{a}, \mathrm{b}$, the addition of a mixture of $\mathrm{CB}[7]$ and $\mathrm{MV}^{2+} 2 \mathrm{I}^{-}$led to a variation in $\zeta$-potential more significantly than separately. To confirm the cooperative effect, two $\zeta-$ potential values were calculated and compared. The first is the change in $\zeta$-potential $\left(\Delta \zeta_{\text {mix }}\right)$ on the addition of the mixture of $\mathrm{CB}[7]$ and $\mathrm{MV}^{2+} 2 \mathrm{I}^{-}$, and the second is the sum in the variations of $\zeta$-potential $\left(\Delta \zeta_{\text {sum }}\right)$ caused solely by $\mathrm{CB}[7]$ and $\mathrm{MV}^{2+} 2 \mathrm{I}^{-}$. The data indicate that the value of $\Delta \zeta_{\text {mix }}$ (Fig. 2c, d, pink column) at different molar ratios of host and guest molecules was ca. 9-58\% higher than that of $\Delta \zeta_{\text {sum }}$ (Fig. 2c, d, orange and green columns), confirming the cooperative effect of $\mathrm{CB}[7]$ and $\mathrm{MV}^{2+} 2 \mathrm{I}^{-}$ in regulating the aggregation of Au NPs.

\section{Cooperative adsorption of counter anions, $\mathrm{MV}^{2+}$, and $\mathrm{CB}$ [7] on surface}

SERS studies at the molecular level revealed that the cooperative effect in aggregating Au NPs originated from the cooperative adsorption of halide counter anions, $\mathrm{MV}^{2+}$, and $\mathrm{CB}[7]$ on the surface of Au NPs. Strong SERS signals of both $\mathrm{MV}^{2+}$ and $\mathrm{CB}[7]$ were observed for the $\mathrm{Au}$ NPs colloid on the addition of a mixture of solutions of $\mathrm{MV}^{2+} 2 \mathrm{I}^{-}$and $\mathrm{CB}[7]$ (Fig. 3a, red line). According to a previous study ${ }^{32}$ and the corresponding ordinary Raman spectra (Fig. S4), the SERS peaks at 838, 1189, 1295, and $1644 \mathrm{~cm}^{-1}$ mainly originated from $\mathrm{C}-\mathrm{C}$ bond stretching, $\mathrm{N}-\mathrm{CH}_{3}$ stretching, inter-ring $\mathrm{C}-\mathrm{C}$ stretching, and ring $\mathrm{C}-\mathrm{C}$ stretching vibration modes of $\mathrm{MV}^{2+}$. The two peaks 

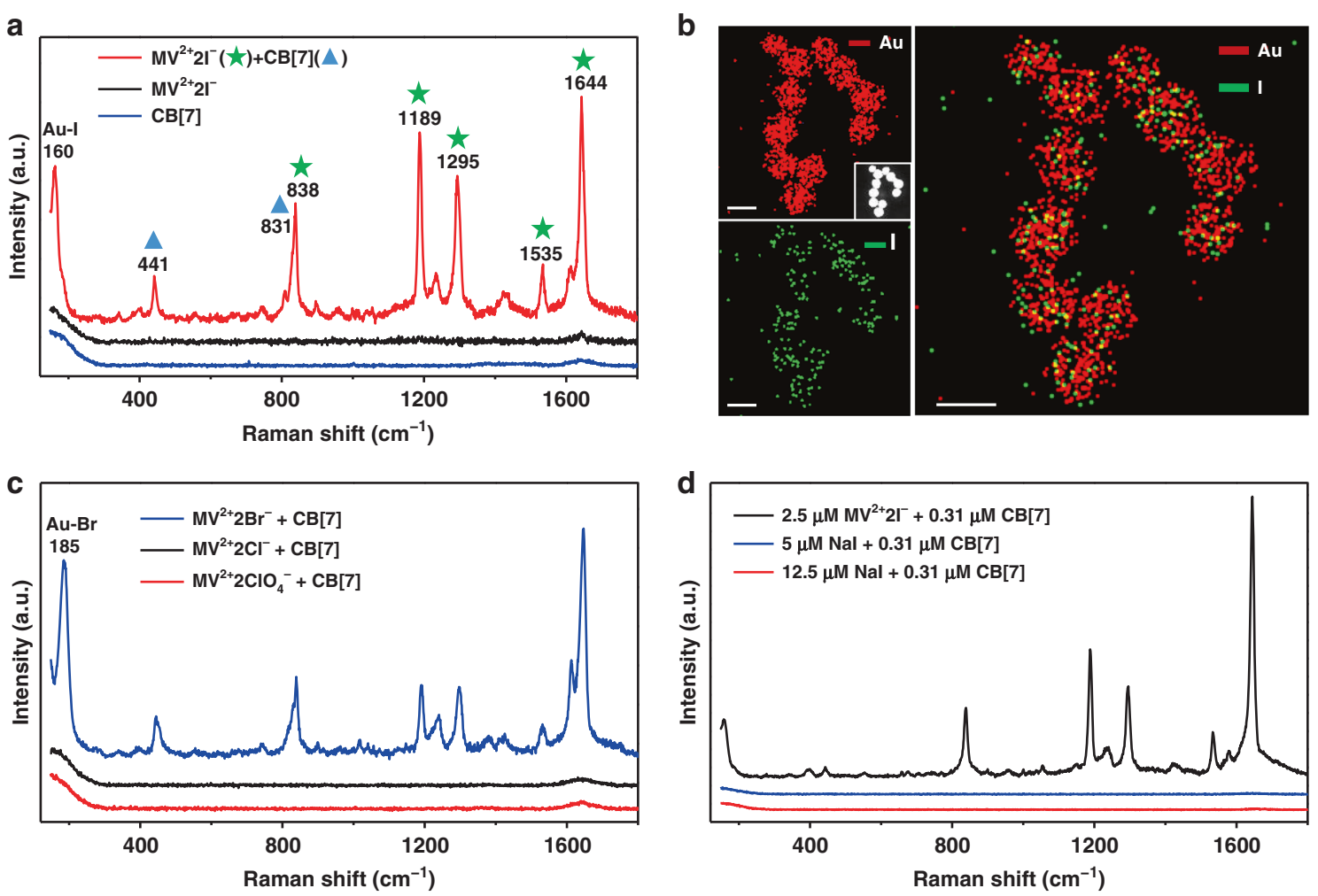

Fig. 3 Cooperative adsorption of counter anions, $\mathbf{M V}^{2+}$, and $\mathbf{C B}[7]$ on Au NPs surface. a SERS spectra of Au NPs colloid on the addition of $\left(\mathrm{MV}^{2+} 2 \mathrm{I}^{-}+\mathrm{CB}[7]\right), \mathrm{MV}^{2+} 2 \mathrm{I}^{-}$, and $\mathrm{CB}[7]$, respectively. The concentrations of $\mathrm{MV}^{2+} 2 \mathrm{I}^{-}$and $\mathrm{CB}[7]$ are $2.5 \mu \mathrm{M}$ and $0.62 \mu \mathrm{M}$, respectively. b STEM elemental mappings of Au NPs aggregates on the addition of $\mathrm{MV}^{2+} 21^{-}(2.5 \mu \mathrm{M})+\mathrm{CB}[7](0.31 \mu \mathrm{M})$. The inset shows the corresponding high-angle annular dark-field image. All the scale bars represent $50 \mathrm{~nm}$. c SERS spectra of Au NPs colloid on the addition of $\mathrm{MV}^{2+} 2 \mathrm{Br}^{-}(2.5 \mu \mathrm{M})+\mathrm{CB}[7](0.31 \mu \mathrm{M})$ and $\mathrm{MV}^{2+} 2 \mathrm{Cl}^{-}(2.5 \mu \mathrm{M})+\mathrm{CB}[7](0.31 \mu \mathrm{M})$, respectively. $\mathbf{d}$ SERS spectra of Au NPs colloid on the addition of different concentrations of CB[7] + $\mathrm{MV}^{2+} 2 \mathrm{I}^{-} / \mathrm{Nal}$

at 441 and $831 \mathrm{~cm}^{-1}$ were assigned to the ring scissor and ring deformation modes of $\mathrm{CB}[\mathrm{n}]$, respectively ${ }^{33}$. Only the in-plane modes of $\mathrm{MV}^{2+}$ and $\mathrm{CB}[7]$ were greatly enhanced, suggesting that they are located on the Au NPs surface with a perpendicular orientation according to the surface selection rules of SERS ${ }^{34-36}$. In addition, the SERS performance was found to be independent of the mixing order of $\mathrm{CB}[7], \mathrm{MV}^{2+} 2 \mathrm{I}^{-}$, and Au NPs colloids, evidenced by the almost identical SERS performance in terms of peak locations and (relative) Raman intensities (Fig. S5). $\mathrm{MV}^{2+} 2 \mathrm{I}^{-}$alone can also induce the aggregation of $\mathrm{Au}$ NPs and thereby produce a SERS signal (Fig. S6), but with 10 times concentration larger than those applied in host-guest induced aggregation (Fig. 3a and Fig. S5).

Interestingly, a strong $\mathrm{Au}-\mathrm{I}$ band $^{37}$ was observed at ca. $160 \mathrm{~cm}^{-1}$ for the $\mathrm{Au}$ NPs colloid on the addition of a mixture of solutions of $\mathrm{MV}^{2+} 2 \mathrm{I}^{-}$and $\mathrm{CB}$ [7], indicating the adsorption of $\mathrm{I}^{-}$, i.e., the counter anions of $\mathrm{MV}^{2+} 2 \mathrm{I}^{-}$, on $\mathrm{Au}$ NPs surface (Fig. 3a). It was further confirmed by the scanning transmission electron microscopy (STEM) elemental mapping of Au NPs aggregates. In the STEM images shown in Fig. 3b, a uniform distribution of $\mathrm{I}^{-}$was observed over Au NPs surface. It has been reported that halide ions can specifically adsorbed on gold surface ${ }^{37,38}$, thereby facilitating the co-adsorption of positively charged molecules through electrostatic interactions ${ }^{39,40}$. Therefore, we conjectured that there exsits the cooperative adsorption among counter anions, $\mathrm{MV}^{2+}$, and $\mathrm{CB}[7]$. Specifically, the halide counter anions are directly adsorbed on $\mathrm{Au}$ NPs surface and attract $\mathrm{MV}^{2+}$; then, $\mathrm{CB}[7]$ interacts with the adsorbed $\mathrm{MV}^{2+}$ to form a sandwich structure.

To verify this conjecture, $\mathrm{I}^{-}$was first replaced with $\mathrm{Br}^{-}$, $\mathrm{Cl}^{-}$, and $\mathrm{ClO}_{4}{ }^{-}$with the binding affinity to Au surface varying in the following order: $\mathrm{I}^{-}>\mathrm{Br}^{-}>\mathrm{Cl}^{-}>>\mathrm{ClO}_{4}{ }^{-37,38}$. As shown in Fig. 3c, the SERS spectrum for $\mathrm{MV}^{2+} 2 \mathrm{Br}^{-}$is similar to the case of $\mathrm{MV}^{2+} 2 \mathrm{I}^{-}$. In comparison, no SERS signals were observed for $\mathrm{MV}^{2+} 2 \mathrm{Cl}^{-}$and $\mathrm{MV}^{2+} 2 \mathrm{ClO}_{4}{ }^{-}$ even at a fourfold increased concentration of guest or host molecules (Fig. S8a). In addition, by replacing $\mathrm{MV}^{2+}$ cations with $\mathrm{Na}^{+}$cations that also bind to $\mathrm{CB}[7]^{41}, \mathrm{Cl}^{-}$ anions still did not work at the same concentration when compared to $\mathrm{I}^{-}$and $\mathrm{Br}^{-}$anions (Fig. S8b). Thus, the adsorption of halide counter anions on the surface played a key role in the aggregation of Au NPs. 


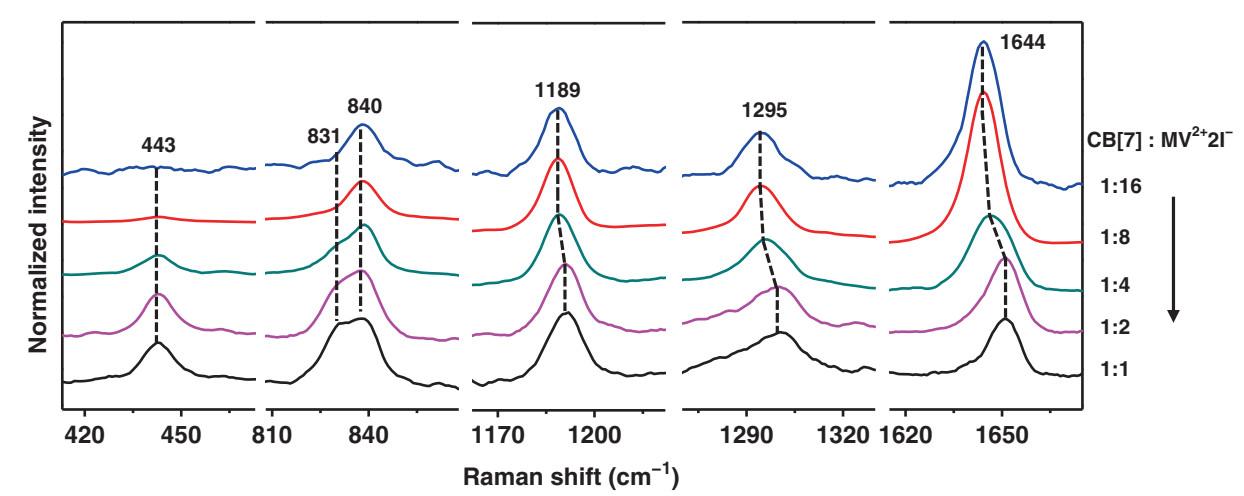

Fig. 4 Normalized SERS spectra (1189 $\mathrm{cm}^{-1}$ peak of $\mathrm{MV}^{2+}$ as standard) in ascending order of molar ratio of $\mathrm{CB}[7]: \mathrm{MV}^{2+} \mathbf{2 I ^ { - }}$ from top to bottom. The concentration of $\mathrm{MV}^{2+} 2 \mathrm{I}^{-}$was kept constant at $2.5 \mu \mathrm{M}$, and that of $\mathrm{CB}[7]$ varied at $0.16 \mu \mathrm{M}, 0.31 \mu \mathrm{M}, 0.62 \mu \mathrm{M}, 1.25 \mu \mathrm{M}$, and $2.5 \mu \mathrm{M}$ from top to bottom

Second, the replacement of $\mathrm{MV}^{2+} 2 \mathrm{I}^{-}$with $\mathrm{NaI}$ induced no SERS signal even at a fourfold increased concentration of $\mathrm{Na}^{+}$and $\mathrm{I}^{-}$up to $12.5 \mu \mathrm{M}$ (Fig. 3d), indicating the key role of $\mathrm{MV}^{2+}$ cations in the aggregation of Au NPs.

\section{Interfacial CB[7]-MV ${ }^{2+}$ complexation}

To further investigate the host-guest complexation at the interface, the CB[7] concentration-dependent SERS experiments were conducted. As shown in Fig. 4, the increase in molar ratio of $\mathrm{CB}[7]: \mathrm{MV}^{2+} 2 \mathrm{I}^{-}$from $1: 8$ to 1:2 led to enhanced Raman signals of $\mathrm{CB}[7]$, including the peaks at $443 \mathrm{~cm}^{-1}$ and ca. $831 \mathrm{~cm}^{-1}$. Besides, the relative intensity of $1644 \mathrm{~cm}^{-1}$ peak from $\mathrm{MV}^{2+}$ decreased significantly. Moreover, the $\mathrm{MV}^{2+}$ peaks at $1189 \mathrm{~cm}^{-1}, 1295 \mathrm{~cm}^{-1}$, and $1644 \mathrm{~cm}^{-1}$ gradually shifted to $1192 \mathrm{~cm}^{-1}, 1301 \mathrm{~cm}^{-1}$, and $1651 \mathrm{~cm}^{-1}$, respectively. Interestingly, such peak shifts were not observed for the same host-guest systems in homogenous solutions (Fig. S4), suggesting the occurrence of a distinct host-guest complexation mechanism on the surface.

To better understand the complexation mechanisms of $\mathrm{CB}[7]$ and $\mathrm{MV}^{2+} 2 \mathrm{I}^{-}$at the interface, control experiments were performed by replacing $\mathrm{CB}[7]$ with other cucurbiturils of smaller cavity sizes, including $\mathrm{CB}[5]$ and $\mathrm{CB}[6]$. Unlike $\mathrm{CB}[7]$, smaller host molecules like $\mathrm{CB}[5]$ and $\mathrm{CB}[6]$ cannot encapsulate bipyridinium owing to their smaller portal diameters and cavity sizes ${ }^{4}$. Hence, they only form exclusion complexes with $\mathrm{MV}^{2+}$ at the portals but not inside the cavities (Fig. S9). Interestingly, both $\mathrm{CB}[5]$ and $\mathrm{CB}[6]$ also cooperatively induced Au NPs aggregation with $\mathrm{MV}^{2+} 2 \mathrm{I}^{-}$(Fig. S10). Moreover, similar blue shifts in the $\mathrm{MV}^{2+}$ peak at $1644 \mathrm{~cm}^{-1}$ were also observed for $\mathrm{CB}[5]$ and $\mathrm{CB}[6]$ systems (Fig. 5). The shifts resulted from the gradual conversion of 1644 to $1651 \mathrm{~cm}^{-1}$ peak, as shown by the Gaussian function fitting. The two peaks were assigned to the adsorbed $\mathrm{MV}^{2+} 2 \mathrm{I}^{-}$complexed without (Fig. S11) or with (Fig. S12) CB[n], respectively. Therefore,

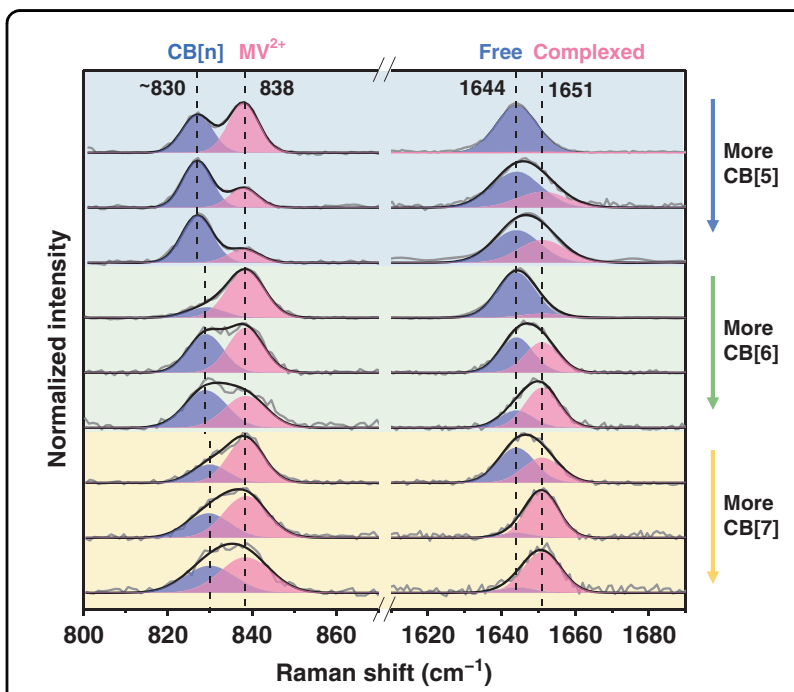

Fig. $5 \mathrm{CB}[5] / \mathrm{CB}[6] / \mathrm{CB}[7]$ concentration-dependent SERS spectra obtained with $\mathbf{2 . 5} \boldsymbol{\mu} \mathbf{M ~ M V}^{\mathbf{2}+} \mathbf{2 I}^{-}$. The concentrations of CB[5]/CB[6]/ $\mathrm{CB}[7]$ are $0.62 \mu \mathrm{M}, 1.25 \mu \mathrm{M}$, and $2.5 \mu \mathrm{M}$ from top to bottom

the complexation model of $\mathrm{CB}[7]$ and $\mathrm{MV}^{2+} 2 \mathrm{I}^{-}$at Au NPs surface may be analogous to that of $\mathrm{CB}[5]$ and $\mathrm{CB}[6]$ systems. In other words, $\mathrm{CB}[7]$ and $\mathrm{MV}^{2+} 2 \mathrm{I}^{-}$formed an exclusion complex on Au NPs surface instead of a conventional 1:1 inclusion complex. Furthermore, the relative Raman intensity of $1651 \mathrm{~cm}^{-1}$ peak over $1644 \mathrm{~cm}^{-1}$ peak under the same conditions increased in the following order: $\mathrm{CB}[5]<\mathrm{CB}[6]<\mathrm{CB}[7]$ system. The quantitative analysis of complexation (Fig. S14) shows a similar 1:2 complexation model between $\mathrm{CB}[5] / \mathrm{CB}[6] / \mathrm{CB}[7]$ and $\mathrm{MV}^{2+} 2 \mathrm{I}^{-}$at the interface, regardless of the changed inner size of $\mathrm{CB}[\mathrm{n}]$, and the obtained interfacial binding constants of host molecules on the surface increase in the following order: $\mathrm{CB}[5]<\mathrm{CB}[6]<\mathrm{CB}[7]$.

Based on these data, an exclusion complexation model between $\mathrm{CB}[7]$ and $\mathrm{MV}^{2+} 2 \mathrm{I}^{-}$at a solid-liquid interface 


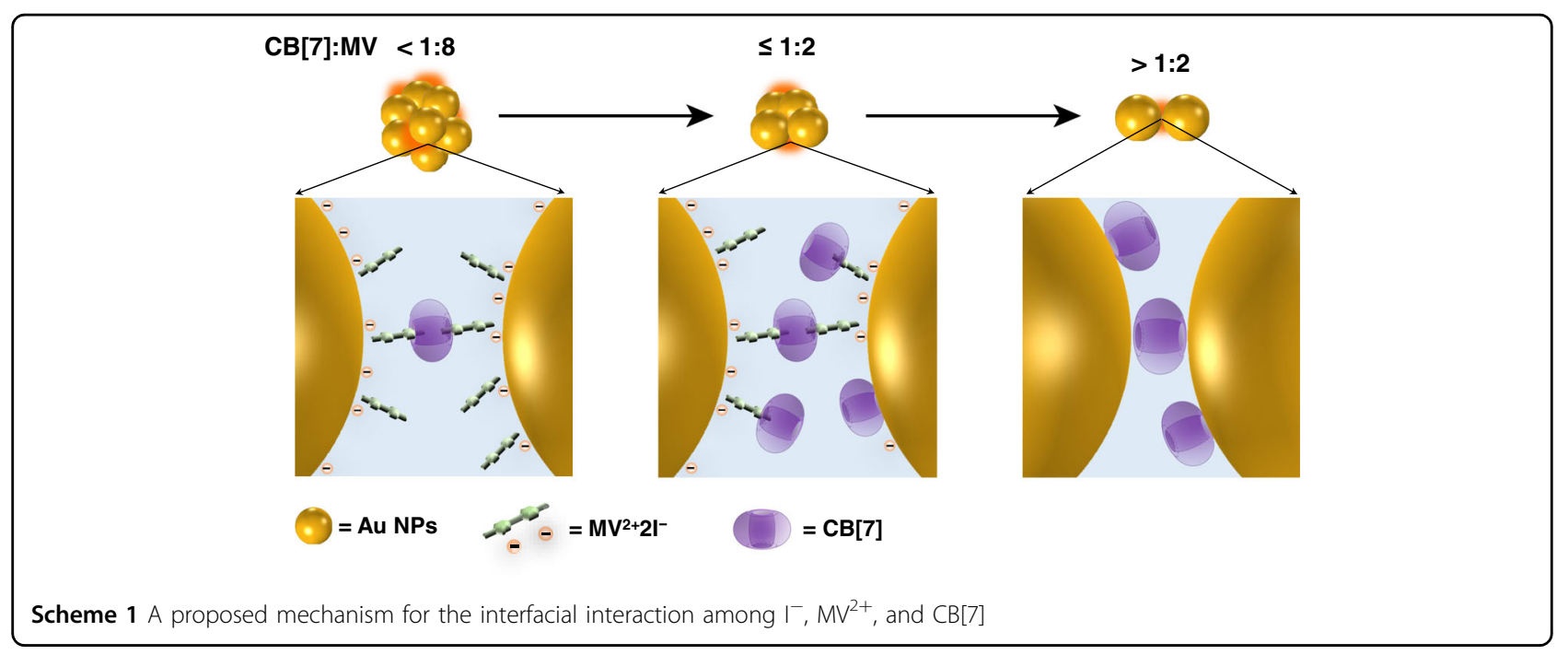

was constructed as shown in Scheme 1. With the aid of electrostatic attraction of adsorbed $\mathrm{I}^{-}$, positively charged $\mathrm{MV}^{2+}$ was adsorbed onto the Au NPs surface in a nearvertical fashion. Meanwhile, the dangling positive $\mathrm{N}$ atom in $\mathrm{MV}^{2+}$ interacts with the portal of $\mathrm{CB}[7]$ and the methyl group is encapsulated in the cavity of $\mathrm{CB}[7]$. This host-guest interaction could be driven by ion-dipole interactions between the positive $\mathrm{N}$ atom and negative portal of $\mathrm{CB}$ [7], as well as the hydrophobic force between methyl group and cavity of $\mathrm{CB}[7]^{29,42,43}$. The interfacial interactions among $\mathrm{Au} \mathrm{NPs}, \mathrm{CB}[7]$, and $\mathrm{MV}^{2+} 2 \mathrm{I}^{-}$were also regulated by the stoichiometry of $\mathrm{CB}[7]$ to $\mathrm{MV}^{2+} 2 \mathrm{I}^{-}$, relevant to $\mathrm{Au}$ NPs aggregation (Figs. S15 and S16). Precisely, a low stoichiometry of $\mathrm{CB}[7]$ (CB[7]: $\left.\mathrm{MV}^{2+} 2 \mathrm{I}^{-} \leq 1: 8\right)$ already caused the intense aggregation of $\mathrm{Au}$ NPs (Scheme 1, left). The Au NPs might be bridged by $\mathrm{CB}[7]-\mathrm{MV}^{2+}$ complexes in the form that one $\mathrm{CB}[7]$ molecule binds two adsorbed $\mathrm{MV}^{2+}$ molecules from adjacent $\mathrm{Au}$ NPs. As the content of $\mathrm{CB}[7]$ increased (1:8 < $\left.\mathrm{CB}[7]: \mathrm{MV}^{2+} 2 \mathrm{I}^{-} \leq 1: 2\right)$, more $\mathrm{CB}[7]$ would bind to the adsorbed $\mathrm{MV}^{2+}$. This results in electrostatic repulsion among Au NPs and partially cleavage of the linkers, thereby reducing the aggregation of Au NPs (Scheme 1, middle). Meanwhile, CB[7] is directly adsorbed on $\mathrm{Au}$ NPs surface and compete against the adsorption of $\mathrm{MV}^{2+} 2 \mathrm{I}^{-}$. Excess $\mathrm{CB}[7]\left(\mathrm{CB}[7]: \mathrm{MV}^{2+} 2 \mathrm{I}^{-}>1: 2\right)$ would completely inhibit the adsorption of $\mathrm{MV}^{2+} 2 \mathrm{I}^{-}$on Au NPs surface, thereby eliminating the cooperative effect between $\mathrm{MV}^{2+} 2 \mathrm{I}^{-}$and $\mathrm{CB}[7]$ and further reducing the aggregation of Au NPs (Scheme 1, right).

\section{Discussion}

The host-guest complexation at the solid-liquid interface is usually assumed to be the same as that in homogeneous solutions. However, synergistic effects among multiple noncovalent interactions based on electrostatic, hydrophilic, and hydrophobic interactions combined with the surface effects like steric hindrance and competitive adsorption ${ }^{5,24}$ greatly affect the host-guest complexation on the surface. Here, the SERS results suggest an unconventional host-guest complexation model on the surface, distinct from the well-known 1:1 inclusion complexation model in aqueous solutions.

Cooperative adsorption of halide counter anions, $\mathrm{MV}^{2+}$, and $\mathrm{CB}[7]$ occurred on the Au NPs surface. The adsorbed $\mathrm{I}^{-}$anions on $\mathrm{Au}$ NPs surface promoted the gathering of $\mathrm{MV}^{2+}$ cations, as well as the host molecules. Under the condition, one positively charged $\mathrm{N}$ atom of $\mathrm{MV}^{2+}$ interacted with $\mathrm{I}^{-}$anions present on the surface, which may impede the encapsulation of bipyridinium unit of $\mathrm{MV}^{2+}$ by $\mathrm{CB}[7]$ owing to the electrostatic repulsion between $\mathrm{I}^{-}$anions and carbonyl oxygens at the $\mathrm{CB}[7]$ portal. DFT calculation (Fig. S17) shows a different profile in the case with $\mathrm{I}^{-}$compared with that without $\mathrm{I}^{-}$; the two local minimums are both exclusive complexation models, suggesting a significant effect of counter anions on interfacial host-guest complexation.

The intrinsic weakness in physical adsorption ${ }^{44}$ led to inevitable competitive adsorption among the host, guest, and counterions on the surface. Competitive adsorption on the surface resulted in diverse surface environments, thereby affecting the host-guest complexation behavior on the surface. Here, the competitive adsorption between $\mathrm{I}^{-}$and $\mathrm{CB}[7]$ on Au NPs surface was clearly characterized by SERS, and it was verified to be closely relevant to the aggregation Au NPs.

In summary, the interfacial interactions of $\mathrm{CB}[\mathrm{n}]$-based host-guest system on Au NPs surfaces were systematically studied by SERS. The ultrahigh sensitivity and rich molecular vibrational information provided by SERS allowed the determination of unreported cooperative adsorptions among counter anions $\left(\mathrm{I}^{-}\right.$and $\left.\mathrm{Br}^{-}\right)$, guest 
cations $\left(\mathrm{MV}^{2+}\right.$ and $\left.\mathrm{Na}^{+}\right)$and hosts $(\mathrm{CB}[5], \mathrm{CB}[6]$, and $\mathrm{CB}$ [7]) on the surface. Moreover, an exclusion complex model between $\mathrm{CB}[7]$ and $\mathrm{MV}^{2+}$ distinct from that in aqueous solutions was proposed based on the SERS results. These findings provide new insights into the fundamental understanding of host-guest interactions at the solid-liquid interface, promising for applications in host-guest chemistry for engineered nanomaterials.

\section{Materials and methods \\ Reagents}

Methyl viologen diiodide $\left(\mathrm{MV}^{2+} 2 \mathrm{I}^{-}\right)$and methyl viologen dichloride $\left(\mathrm{MV}^{2+} 2 \mathrm{Cl}^{-}\right)$were purchased from $\mathrm{J} \& \mathrm{~K}$ Chemical. CB[7], CB[6], and sodium 3-(trimethylsilyl)-1propanesulfonate $\left[{ }^{1} \mathrm{H}\right.$ NMR standard used for $\mathrm{D}_{2} \mathrm{O}$ solvent] were obtained from Sigma-Aldrich. Methyl viologen dibromide $\left(\mathrm{MV}^{2+} 2 \mathrm{Br}^{-}\right)$was synthesized following literature $^{45}$. High-purity water (Milli-Q, $18.2 \mathrm{M} \Omega \mathrm{cm}$ ) was used throughout the studies.

\section{Preparation of Au NPs}

In all, $50 \mathrm{~nm} \mathrm{Au}$ NPs were prepared using the citrate reduction method reported by Lee and Meisel ${ }^{46}$. To concentrate the Au NPs by 10 times, $14 \mathrm{~mL}$ colloid suspension was centrifuged ( $3500 \mathrm{rpm}, 15 \mathrm{~min}$ ) once and resuspended in $1.4 \mathrm{~mL} \mathrm{H}_{2} \mathrm{O}$ after the removal of all the supernatant.

\section{Preparation of stock solutions}

All stock solutions $\left(2 \mathrm{mM}\right.$ ) were prepared in $4 \mathrm{~mL} \mathrm{H}_{2} \mathrm{O}$ followed by dilution to $50 \mu \mathrm{M}$ and then to $10 \mu \mathrm{M}$.

\section{UV-Vis spectroscopy}

The ultraviolet-visible (UV-vis) spectra were recorded using a Shimada UV-2550 spectrophotometer. In all, $100 \mu \mathrm{L} \mathrm{Au} \mathrm{NPs} \mathrm{and} 300 \mu \mathrm{L}$ of reagents were mixed and immediately placed in a cuvette with a one-millimeter optical path. The time-dependent spectra were acquired at $1 \mathrm{~min}$ intervals for $5 \mathrm{~min}$.

\section{$\zeta$-potential measurements}

The $\zeta$-potential was collected using a Malvern Zetasizer Nano ZS Instrument. First, $200 \mu \mathrm{L}$ Au NPs and $600 \mu \mathrm{L}$ reagents were mixed and then immediately placed into a cuvette. The temperature was set as $25^{\circ} \mathrm{C}$, and every sample was tested three times.

\section{SERS experiments}

The SERS data were collected using a Renishaw Invia instrument $(5 \mathrm{~mW}, 633 \mathrm{~nm}, \times 50$ objective, $\mathrm{NA}=0.55$, $1800 \mathrm{gr} / \mathrm{mm}$ grating, and $20 \mathrm{~s}$ per scan). First, calculated volumes of stock solutions were mixed in a 96-well plate, and then $50 \mu \mathrm{L}$ of concentrated Au NPs was added and mixed through pipetting a dozen times. The total volume was adjusted to $200 \mu \mathrm{L}$, and SERS measurements were immediately carried out after mixing by focusing the laser beam directly on the sample suspension.

\section{Transmission electron microscopy}

TEM images were viewed using a JEM-1400 (JEOL, Japan) and scanning TEM images were collected using a Talos F200 instrument (FEI, USA).

\section{Nuclear magnetic resonance}

NMR spectra were collected using a Bruker AVANCE III-500 MHz NMR unless otherwise stated.

\section{Acknowledgements}

This work was financially supported by the NSFC (no. 41876099, 91427304, 91227111, 21722304, and 21971216), and the Xiamen Science and Technology Project (no. 3502Z20183002). We also thank Liubin Feng, Gen Li, and Ze-Zhong Xie for their assistance with the experiments.

\section{Author details}

${ }^{1}$ State Key Laboratory of Physical Chemistry of Solid Surfaces, Collaborative Innovation Center of Chemistry for Energy Materials (iChEM), College of Chemistry and Chemical Engineering, Xiamen University, Xiamen 361005, China. ${ }^{2}$ State Key Laboratory of Marine Environmental Science, Fujian Provincial Key Laboratory for Coastal Ecology and Environmental Studies, Center for Marine Environmental Chemistry \& Toxicology, College of the Environment and Ecology, Xiamen University, Xiamen 361102, China. ${ }^{3}$ School of Electronic Science and Engineering (National Model Microelectronics College), Xiamen University, Xiamen 361005, China. ${ }^{4}$ Key Laboratory of Chemical Biology of Fujian Province, Xiamen University, Xiamen 361005, China

\section{Author contributions}

The manuscript was written through the contributions of all authors. All authors have given approval to the final version of the manuscript.

Conflict of interest

The authors declare no competing interests.

Supplementary information The online version contains supplementary material available at https://doi.org/10.1038/s41377-021-00526-5.

Received: 28 October 2020 Revised: 20 March 2021 Accepted: 31 March 2021

Published online: 19 April 2021

References

1. Murray, J. et al. The aqueous supramolecular chemistry of cucurbit[n]urils, pillar[n]arenes and deep-cavity cavitands. Chem. Soc. Rev. 46, 2479-2496 (2017).

2. Liu, Z. C., Nalluri, S. K. M. \& Stoddart, J. F. Surveying macrocyclic chemistry: from flexible crown ethers to rigid cyclophanes. Chem. Soc. Rev. 46, 2459-2478 (2017).

3. Yu, G. C., Jie, K. C. \& Huang, F. H. Supramolecular amphiphiles based on host-guest molecular recognition motifs. Chem. Rev. 115, 7240-7303 (2015).

4. Barrow, S. J. et al. Cucurbituril-based molecular recognition. Chem. Rev. 115, 12320-12406 (2015)

5. Descalzo, A. B. et al. The supramolecular chemistry of organic-inorganic hybrid materials. Angew. Chem. Int. Ed. 45, 5924-5948 (2006).

6. Yang, $H$. et al. Supramolecular chemistry at interfaces: host-guest interactions for fabricating multifunctional biointerfaces. Acc. Chem. Res. 47, 2106-2115 (2014).

7. Kim, H. J. et al. Host-guest sensing by calixarenes on the surfaces. Chem. Soc. Rev. 41, 1173-1190 (2012).

8. Ma, X. \& Zhao, Y. L. Biomedical applications of supramolecular systems based on host-guest interactions. Chem. Rev. 115, 7794-7839 (2015). 
9. Montes-García, V. et al. Metal nanoparticles and supramolecular macrocycles: a tale of synergy. Chem. Eur. J. 20, 10874-10883 (2014).

10. Pazos, E. et al. Cucurbit[8]uril (CB[8])-based supramolecular switches. Angew. Chem. Int. Ed. 58, 403-416 (2019)

11. Lou, X. Y. \& Yang, Y. W. Pillar[n]arene-based supramolecular switches in solution and on surfaces. Adv. Mater. 32, 2003263 (2020).

12. Tan, L. L. et al. Cucurbiturils-mediated noble metal nanoparticles for applications in sensing, sers, theranostics, and catalysis. Adv. Funct. Mater. 31, 2007277 (2020).

13. Kasera, S. et al. Quantitative SERS using the sequestration of small molecules inside precise plasmonic nanoconstructs. Nano Lett. 12, 5924-5928 (2012).

14. Montalti, M. et al. Solvent-induced modulation of collective photophysical processes in fluorescent silica nanoparticles. J. Am. Chem. Soc. 124 13540-13546 (2002)

15. Basabe-Desmonts, L. et al. A simple approach to sensor discovery and fabrication on self-assembled monolayers on glass. J. Am. Chem. Soc. 126, 7293-7299 (2004).

16. Zheng, Y. J. et al. Development of fluorescent film sensors for the detection of divalent copper. J. Am. Chem. Soc. 125, 2680-2686 (2003).

17. Labande, A., Ruiz, J. \& Astruc, D. Supramolecular gold nanoparticles for the redox recognition of oxoanions: syntheses, titrations, stereoelectronic effects, and selectivity. J. Am. Chem. Soc. 124, 1782-1789 (2002).

18. Astruc, D., Daniel, M. C. \& Ruiz, J. Dendrimers and gold nanoparticles as exoreceptors sensing biologically important anions. Chem. Commun. 2367-2649 (2004).

19. Liu, X. et al. Determination of monolayer-protected gold nanoparticle ligandshell morphology using NMR. Nat. Commun. 3, 1182 (2012).

20. Smith, A. M. et al. Quantitative analysis of thiolated ligand exchange on gold nanoparticles monitored by $1 \mathrm{H}$ NMR spectroscopy. Anal. Chem. 87, 2771-2778 (2015).

21. Anderson, N. C. \& Owen, J. S. Soluble, chloride-terminated CdSe nanocrystals: ligand exchange monitored by ${ }^{1} \mathrm{H}$ and ${ }^{31} \mathrm{P}$ NMR spectroscopy. Chem. Mater. 25, 69-76 (2012).

22. Langer, J. et al. Present and future of surface-enhanced Raman scattering. ACS Nano 14, 28-117 (2020)

23. Novo, C., Funston, A. M. \& Mulvaney, P. Direct observation of chemical reactions on single gold nanocrystals using surface plasmon spectroscopy. Nat. Nanotechnol. 3, 598-602 (2008).

24. De Nijs, B. et al. Inhibiting analyte theft in surface-enhanced raman spectroscopy substrates: subnanomolar quantitative drug detection. ACS Sens. 4 2988-2996 (2019).

25. Sigle, D. O. et al. Observing single molecules complexing with cucurbit[7]uril through nanogap surface-enhanced raman spectroscopy. J. Phys. Chem. Lett. 7, 704-710 (2016)

26. Leyton, P. et al. Surface-enhanced micro-raman detection and characterization of calix[4]arene-polycyclic aromatic hydrocarbon host-guest complexes. Appl. Spectrosc. 59, 1009-1015 (2005).

27. Leyton, P. et al. Selective molecular recognition of polycyclic aromatic hydrocarbons (PAHs) on calix[4]arene-functionalized Ag nanoparticles by surface-enhanced Raman scattering. J. Phys. Chem. B 108, 17484-17490 (2004)
28. Ong, W., Gómez-Kaifer, M. \& Kaifer, A. E. Cucurbit[7]uril: a very effective host for viologens and their cation radicals. Org. Lett. 4, 1791-1794 (2002).

29. Moon, K. \& Kaifer, A. E. Modes of binding interaction between viologen guests and the cucurbit[7]uril host. Org. Lett. 6, 185-188 (2004).

30. Lee, T. C. \& Scherman, O. A. Formation of dynamic aggregates in water by cucurbit[5]uril capped with gold nanoparticles. Chem. Commun. 46 , 2438-2440 (2010)

31. Taylor, R. W. et al. Precise subnanometer plasmonic junctions for SERS within gold nanoparticle assemblies using cucurbit[n]uril "glue". ACS Nano 5, 3878-3887 (2011)

32. Castellà-Ventura, M. \& Kassab, E. Vibrational analysis of some transient species implicated in the photoreduction of 4, 4'-bipyridine based on ab initio and density functional calculations. J. Raman Spectrosc. 29, 511-536 (1998).

33. Mahajan, S. et al. Raman and SERS spectroscopy of cucurbit[n]urils. Phys. Chem Chem. Phys. 12, 10429-10433 (2010).

34. Joo, S. W. Surface-enhanced Raman scattering of 4, 4'-bipyridine on gold nanoparticle surfaces. Vib. Spectrosc. 34, 269-272 (2004).

35. Pérez-Jiménez, A. I. et al. Surface-enhanced Raman spectroscopy: benefits, trade-offs and future developments. Chem. Sci. 11, 4563-4577 (2020).

36. Roldán, M. L. et al. Cucurbit[8]uril-stabilized charge transfer complexes with diquat driven by pH: a SERS study. Phys. Chem. Chem. Phys. 14, 4935-4941 (2012).

37. Gao, P. \& Weaver, M. J. Metal-adsorbate vibrational frequencies as a probe of surface bonding: halides and pseudohalides at gold electrodes. J. Phys. Chem. 90, 4057-4063 (1986)

38. Ignaczak, A. \& Gomes, J. A. N. F. Quantum calculations on the adsorption of halide ions on the noble metals. J. Electroanal. Chem. 420, 71-78 (1997).

39. Lopez-Ramirez, M. R. et al. Vibrational analysis of herbicide diquat: a normal Raman and SERS study on Ag nanoparticles. Vib. Spectrosc. 48, 58-64 (2008).

40. Leopold, N. et al. The role of adatoms in chloride-activated colloidal silver nanoparticles for surface-enhanced Raman scattering enhancement. Beilstein J. Nanotechnol. 9, 2236-2247 (2018).

41. Khan, M. S. A. et al. Remarkable salt effect on stability of supramolecular complex between modified cucurbit[6]uril and methylviologen in aqueous media. J. Phys. Chem. B 113, 11054-11057 (2009).

42. Danylyuk, O., Fedin, V. P. \& Sashuk, V. Kinetic trapping of the host-quest association intermediate and its transformation into a thermodynamic inclusion complex. Chem. Commun. 49, 1859-1861 (2013).

43. Vincil, G. A. \& Urbach, A. R. Effects of the number and placement of positive charges on viologen-cucurbit[n] uril interactions. Supramol. Chem. 20, 681-687 (2008).

44. Bell, S. E. \& Sirimuthu, N. M. S. Surface-enhanced Raman spectroscopy as a probe of competitive binding by anions to citrate-reduced silver colloids. J. Phys. Chem. A 109, 7405-7410 (2005).

45. Bhowmik, P. K. et al. Synthesis and characterization of ionic liquids: viologen bis\{tetrakis[3, 5-bis(trifluoromethyl)phenyl]borate\} salts. Liq. Cryst. 33, 891-906 (2006).

46. Lee, P. C. \& Meisel, D. Adsorption and surface-enhanced raman of dyes on silver and gold sols. J. Phys. Chem. 86, 3391-3395 (1982). 